Vorwort

Marina Münkler*

\title{
Einige Grundüberlegungen zum Konzept und zur Reichweite invektiver Gattungen
}


Der vorliegende Band geht auf eine Tagung zurück, die das Teilprojekt E des Sonderforschungsbereichs 1285 Invektivität. Konstellationen und Dynamiken der Herabsetzung unter dem Titel Invektive Gattungen. Formen und Medien der Herabsetzung vom 19.-21. Februar 2020 in Dresden veranstaltet hat. ${ }^{1}$ Die Tagung ging von der Frage nach der Funktion von Gattungen für invektive Kommunikationen aus und thematisierte damit eines der zentralen Problemfelder des SFB. Grundlegend begreift das Konzept der ,Invektivität' Phänomene der Schmähung und Herabwürdigung als epochen- und kulturübergreifende Arten von Kommunikation, die als Störungs- und Dynamisierungs-, aber auch als Stabilisierungsmomente gesellschaftliche Ordnungen prägen und das Potenzial besitzen, Gemeinschaften zu verändern oder zu zerstören, durch Ab- und Ausgrenzung, aber auch zu bilden oder zu stabilisieren. $^{2}$ Der Terminus der Invektivität bezeichnet jene verbalen und nonverbalen, schriftlichen und mündlichen, aber auch bildlichen und medialen Aspekte von Kommunikation, mittels derer Personen oder Gruppen ausgegrenzt, herabgesetzt, stigmatisiert, beschämt, gekränkt oder lächerlich gemacht werden können.

Entgegen dem mitunter ersten Eindruck entfaltet Invektivität ihr herabsetzendes Potenzial nicht einfach in spontanen Akten, sondern zumeist in mehr oder minder stabilen Mustern. Solche

1 Vgl. Michalsky, Sophia/Haugk, Theresa: Tagungsbericht: Invektive Gattungen. Formen und Medien der Herabsetzung. Interdisziplinäre Tagung des TP E „Sakralität und Sakrileg. Die Herabsetzung des Heiligen im interkonfessionellen Streit des 16. Jahrhunderts". 19.02.2020-21.02.2020. Dresden. In: H-Soz-Kult, 23.04.2020. URL: https://www. hsozkult.de/conferencereport/id/tagungsberichte-8733 (letzter Zugriff: 01.11.2021).

2 Vgl. Ellerbrock/Koch/Müller-Mall et al. (2017) Invektivität.
Muster können durch rhetorische und literarische Gattungen bereitgestellt, durch kommunikative Gattungen verfestigt, aber auch durch mediale Affordanzen initiiert werden. Die Erscheinungsformen und Funktionen des Invektiven treten in sozialer, politischer, medialer und ästhetischer Hinsicht jedoch in vielschichtigen, historisch variablen Konstellationen auf und lassen sich nicht immer problemlos zuordnen. Unter dem Aspekt dieser komplexen Dynamik gehen wir davon aus, dass das Formenspektrum der Invektivität mit traditionellen Gattungstaxonomien nicht hinreichend präzise beschrieben ist. ${ }^{3}$ Wie die Beiträge des Bandes zeigen, erweisen sich die Modi und Modalitäten des Invektiven einerseits als iterativ, andererseits als ausgesprochen fluide und transformierbar, da sie abhängig von den sie prägenden Dispositiven, Konstellationen und sozialen Positionierungen an unterschiedliche kommunikative Situationen angepasst werden, sodass sie in keiner Liste von invektiven rhetorischen oder literarischen Gattungen aufgehen. Das Feld des Invektiven umfasst überdies auch alltagssprachliche Sprech- und Schreibweisen, die als kommunikative Gattungen verstanden werden können und nicht mit den gängigen literaturwissenschaftlichen Systematiken zu erfassen sind.

Grundsätzlich stellt sich daher die Frage, was Gattungsbegriffe für die Beschreibung invektiver Modalitäten von Kommunikation leisten können und wie sie gattungstheoretisch elaboriert formuliert werden müssen. Normativ-taxonomische literarische Gattungsbegriffe, die sich an der herkömmlichen Trias von Lyrik, Epik und Dramatik orientieren, können zwar in vielerlei Hinsicht als überholt gelten, spielen aber in anthropologisch

3 Zu grundsätzlichen Aspekten der Gattungsdynamik vgl. Zymner (2007) Gattungsvervielfältigung. 
orientierten Ansätzen nach wie vor eine Rolle. ${ }^{4}$ Für die Beschreibung invektiver Gattungen sind sie freilich zu undifferenziert und überdies als anthropologisch rückgebundene Kategorien zu statisch konzipiert. Geeigneter für die Beschreibung dynamischer Transformationen erscheinen dagegen funktionsgeschichtlich ausgerichtete Gattungskonzepte, die das Verhältnis zwischen der Sozialabhängigkeit und Zweckbedingtheit von Gattungen betonen und sie als "soziokulturelle Konventionen" begreifen. ${ }^{5}$ Demnach können sich Muster auskristallisieren, stabilisieren und institutionell festigen, die dann als gattungshaft dominante Strukturen beschrieben werden können, wenn die sozialen und funktionalen Bedürfnisse innerhalb des Literatursystems dies ermöglichen. Das heißt nicht, dass Gattungen als unmittelbare Antworten auf gesellschaftliche Probleme beschrieben würden, aber funktionsgeschichtlich orientierte Gattungsbegriffe gehen davon aus, dass literarische Gattungen innerhalb des Systems der Literatur - mit Niklas Luhmann gesprochen - Aspekte der sozialen Umwelt in ihre Autopoiesis einbauen. ${ }^{6}$ Dieser funktionsgeschichtliche Ansatz kann durch den rezeptionsästhetischen Zugang ergänzt werden, wonach Textreihen ab einem bestimmten Grad der Verfestigung Erwartungshorizonte prägen, d. h. Erwartungserwartungen aufbauen, die sowohl auf der Produzent:innen- als auch auf der Rezipient:innenseite wirken. ${ }^{7}$ In eine ähnliche Richtung weist der von Gérard Genette eingeführte Begriff der Architextualität. ${ }^{8}$ Wie seine Untersuchung von Formen der Transtextualität gezeigt hat, sind für die Ausprägung von Gattungen nicht zuletzt Paratexte relevant, die - etwa in Titeln und Untertiteln - Zuordnungen von Texten vornehmen und damit einen Text in bestimmter Weise typisieren, indem sie inn etwa als Satire ausweisen und damit bestimmte Erwartungshorizonte erzeugen. ${ }^{9}$ Über solche Typisierungsfunktionen hinaus können Paratexte, wie Widmungsschreiben, Vorreden,

4 Zur Reflexion solcher Gattungsbegriffe vgl. Zymner (2003) Gattungstheorie; Hempfer (1973) Gattungstheorie; Zur Geschichte von Gattungsbegriffen vgl. Gymnich (2010) Gattung.

5 Vgl. Voßkamp (1977) Gattungen, S. 253.

6 Vgl. Voßkamp (1977) Gattungen, S. $258 f$.

7 Vgl. Jauß (1977) Theorie der Gattungen.

8 Vgl. Genette (1993) Palimpseste, S. 14.

9 Vgl. Genette (2001) Paratexte.
Vorworte und Nachworte, aber auch Orte der Gattungsdiskussion sein, an denen Transformationen und Neubestimmungen von Gattungskonventionen und -erwartungen oder Abwertungen etablierter Gattungen vorgenommen werden, wie Antje Sablotny dies in ihrem Beitrag am Beispiel der invektiven Transformation der Legende in die aus einem herabsetzenden Wortspiel hervorgegangene Lügende zeigt. ${ }^{10}$

Die rezeptionsästhetischen Gattungstheorien sind durch praxeologische Ansätze erweitert und stellenweise abgelöst worden, die sich unter dem Oberbegriff von ,Doing Genre' als diskursive Prozeduren der Herstellung von Gattungszuordnungen beschreiben lassen. Gattungen bilden sich nach den praxeologischen Ansätzen nicht ausschließlich und nicht einmal in erster Linie durch die Orientierung an vorgängigen Texten oder einem Prototypen, sondern durch iterative Bezeichnungen von kommunikativen Akten und deren Typisierung als Gattung. ${ }^{11}$ Solche Typisierungen erweisen sich als gattungsprägend, weil sie mit der Zuordnung zugleich Erwartungshorizonte erzeugen, wie Simon Meier-Vieracker in seinem Beitrag zur Wutrede demonstriert.

Funktionsgeschichtliche und praxeologische Ansätze zur Beschreibung literarischer Gattungen können nicht zuletzt auf die rhetorischen Gattungen bezogen werden, die bereits in der Antike von einer Redepraxis und ihrer Funktion sowie deren sozialer Situierung ausgegangen sind. Die grundlegende Funktion der Rede besteht nach der antiken Rhetorik darin, den Zuhörer für die vorgetragene Überzeugung des Redners zu gewinnen. Diese Funktion kann sowohl argumentativ als auch affektiv erfüllt werden: Zu den argumentativen Mitteln gehören belehren (docere) und beweisen (probare), zu den affektiven, die noch einmal nach den besänftigenden und den erregenden Modalitäten unterteilt werden, gewinnen (conciliare), erfreuen (delectare), bewegen (movere) und anstacheln (concitare), deren Wirkungsästhetik mit Ethos und Pathos verknüpft sind. ${ }^{12}$ Die Zuhörenden sollen von der Schuld oder Unschuld eines Angeklagten überzeugt, für eine

10 Siehe den Beitrag von Antje Sablotny in diesem Band. Vgl. daneben auch: Sablotny (2019) Metalegende, bes. S. 166f. sowie Münkler (2015) Legende/Lügende.

11 Vgl. Meier/Marx (2019) Doing Genre.

12 Vgl. Schulz (2019) Was ist rhetorische Wirkung. 
politische Haltung oder Vorgehensweise eingenommen werden, einem Lob oder einer Schmähung zustimmen. Aus diesen Funktionen sind in der antiken Rhetorik schon sehr früh Gattungsdefinitionen hervorgegangen, die ebenfalls eine Gattungstrias erzeugten. Nach den Typisierungen der Rhetoriklehrbücher seit Aristoteles wurden sie traditionell über ihre institutionelle Verortung, ihre Funktion oder ihre Modalität definiert: die Gerichtsrede (genus iudicale), die Beratungsrede oder politische Rede (genus deliberativum) sowie die Lobrede (genus demonstrativum). ${ }^{13}$ Die funktionale Orientierung und die Ausdifferenzierung nach institutionellen Orten, Anlässen und Modalitäten ermöglichte für die rhetorischen Gattungen jedoch ein enorm flexibles Gattungskonzept, das Anpassungen und Transformationen jederzeit erlaubte. Das zeigt sich nicht zuletzt an der Schmährede (४óyos/vituperatio), die von der Lobrede (ع̈naıvoc/laus) als deren tadelndes Pendant abgeleitet worden ist, indem die Funktion des Lobens durch die des Tadelns ersetzt wurde. Freilich kann die Funktion des Tadelns in sehr unterschiedlicher Weise wahrgenommen werden, die vom freundlich-kritischen Tadel bis zur sozial vernichtenden Schmähung reicht, für die sich seit der Spätantike die Bezeichnung invectiva oratio etabliert hat. ${ }^{14}$ Wie Dennis Pausch in seinem Beitrag zeigt, erwies sich für die vituperatio aber deren Lehrbarkeit im Rahmen der rhetorischen Gattungslehre als ein Problem, denn die Erwartbarkeit einer Schmähung minderte deren Wirkung und konnte zudem auf den Schmähenden zurückfallen, wenn sie nicht als spontane, sondern als geplante Herabsetzung identifiziert wurde.

Etablierte Muster bilden sich nicht nur in literarischen und rhetorischen Gattungen aus, sondern auch innerhalb der alltäglichen mündlichen Kommunikation. Solche Muster sind zunächst in der Soziologie und daran anschließend in der Linguistik als kommunikative Gattungen beschrieben worden. Der von Thomas Luckmann konzipierte und von Susanne Günthner und Hubert Knoblauch weiterentwickelte Begriff der kommunikativen Gattung beschreibt konventionalisierte kommunikative Vorgänge, die sich zur Bewäl-

13 Vgl. Erler/Tornau (2019) Antike Rhetorik.

14 Siehe dazu den Beitrag von Dennis Pausch in diesem Band. Vgl. daneben Koster (1980) Invektive; Helmrath (2010) Streitkultur. tigung wiederkehrender kommunikativer Probleme oder Situationen etabliert haben. ${ }^{15}$ Damit erleichtern kommunikative Gattungen gesellschaftliches Handeln, da sie für bestimmte Redesituationen und Funktionen verfügbare Muster für Sprachhandlungen anbieten. Günthner und Knoblauch deuten die kommunikativen Gattungen von daher als "Verbindungselement zwischen dem subjektiven Wissensvorrat und den sozialen Strukturen einer Gesellschaft". ${ }^{16}$ Zur Beschreibung kommunikativer Gattungen unterscheiden Luckmann, Günthner und Knoblauch zwischen ihrer Binnenstruktur, der Außenstruktur und der situativen Realisierungsebene von kommunikativen Gattungen. ${ }^{17}$ Die Binnenstruktur umfasst die verbalen sowie die nonverbalen Anteile des kommunikativen Geschehens wie lexiko-semantische und morphologisch-syntaktische Elemente sowie Mimik und Gestik; die Außenstruktur wird vom sozialen Umfeld gebildet, wozu Institutionen (etwa die Schule), Geschlechterkonstellationen (z. B. Gespräche unter Männern) und soziale Gruppen (Handwerker:innen) gehören; zur situativen Realisierungsebene gehören etwa die Themenorientierung, die Zuteilung des Rederechts, Sprecherwechsel etc.

Solche Muster sind nicht nur für die Alltagskommunikation und die in ihr wiederkehrenden Vorgänge höchst relevant, sondern auch für spezifische kommunikative Situationen innerhalb von Institutionen, wie etwa der Schule. Auch hier bieten sich zahlreiche Anknüpfungspunkte für invektivitätstheoretische Fragestellungen: Welche spezifischen invektiven kommunikativen Gattungen sich etwa in Orientierungskursen für Geflüchtete ausbilden, deren Integration in erster Linie durch die Distanzierung von den ihnen unterstellten Mentalitäten ihrer Herkunftskulturen erreicht werden soll, zeigt der Beitrag von Heike Greschke und Youmna Fouad in diesem Band.

Sind Luckmanns Untersuchungen zunächst von unmittelbaren Interaktionssituationen ausgegangen, wie sie für die Schule kennzeichnend

15 Vgl. Luckmann (1986) Kommunikative Gattungen; Günthner/Knoblauch (1994) „Forms are the Food of Faith"; Günthner/Knoblauch (1996) Analyse kommunikativer Gattungen.

16 Günthner/Knoblauch (1994) „Forms are the Food of Faith", S. $715 f$.

17 Vgl. Günthner/Knoblauch (1994) „Forms are the Food of Faith", S. 704ff. 
sind, so ist das Konzept der kommunikativen Gattungen schon bald auf größere Textkorpora sowie auf die Kommunikationen in audio-visuellen Medien übertragen worden. Noah Bubenhofer hat das Konzept der an der mündlichen Sprache orientierten kommunikativen Gattungen auf die korpusbasierte quantitative Untersuchung von Texten angewendet und sie mit dem Begriff der Sprachgebrauchsmuster fruchtbar zu machen versucht. ${ }^{18}$ Dazu müssen bestimmte Aspekte der Binnenstruktur wie Mimik und Gestik ausgeblendet und der Fokus auf lexiko-semantische, morpho-syntaktische und stilistische Merkmale gerichtet werden. Daneben hat sich das Konzept der kommunikativen Gattungen oder Sprachgebrauchsmuster insbesondere zur Beschreibung von spezifischen kommunikativen Formaten des Internets wie dem Blog und dem Chat etabliert. ${ }^{19}$ Schon an diesen kommunikativen Formaten zeigt sich, dass Medien kommunikative Gattungen nicht nur aufnehmen, sondern aufgrund ihrer Affordanzstruktur auch hervorbringen. Die Untersuchung medialer Affordanzen, die zunächst in der Mediensoziologie entwickelt worden ist, konzentriert sich auf die Möglichkeits- und Gelegenheitsstrukturen von Medien und Plattformen, die bestimmte Kommunikationsformen und Gattungen präformieren, wie Katja Kanzler am Beispiel US-amerikanischer populärer Fernsehformate demonstriert. ${ }^{20}$ Sie lässt sich aber auch auf den Medienwandel der Frühen Neuzeit anwenden, wie Albrecht Dröse in seinem Beitrag zur Flugschrift in diesem Band darlegt.

Daher stellt sich die Frage, in welchen kommunikativen Formen und Mustern, literarischen und rhetorischen Gattungen sich Invektivität realisiert und welche medialen Affordanzen invektive Kommunikationsformen initiieren, situieren und etablieren. Zweifellos einschlägig sind rhetorische und literarische Gattungen, die per se als herabsetzend

$18 \mathrm{Vgl}$. Bubenhofer (2009) Sprachgebrauchsmuster, bes. S. $58-60$.

19 Vgl. Dürscheid (2013); Dürscheid/Frick (2016) Schreiben digital, bes. S. 109-128; Ayaß (2011) Kommunikative Gattungen; Lomborg (2011).

$20 \mathrm{Vgl}$. Fox/Panagiotopoulos/Tsouparopoulou (2015) Affordanz; Zillien (2008) Die (Wieder-)Entdeckung der Medien, bes. S. 165-171. gelten, wie Satire ${ }^{21}$, Polemik ${ }^{22}$, Pamphlet ${ }^{23}$ oder Pasquill ${ }^{24}$. Bezeichnend ist allerdings, dass diese terminologischen Zuschreibungen in ihrem Inhalt und Umfang umso unschärfer erscheinen, je langlebiger die jeweiligen Gattungen sind. Während es sich beim Pasquill, wie Gerd Schwerhoff in seinem Beitrag verdeutlicht, um eine kurzlebige Gattungsbezeichnung der Frühen Neuzeit handelt, ist die Gattungsbezeichnung Satire von ungebrochener Aktualität. Allerdings ist möglicherweise gerade deshalb umstritten, ob sie überhaupt angemessen als Gattung beschrieben werden kann oder ob es sich vielmehr um eine Schreibweise handelt. Auch die Charakterisierung als Schreibweise greift indes zu kurz, da Satirisches nicht unbedingt auf Schriftlichkeit angewiesen ist, sondern sich auch bildlich in der Karikatur ${ }^{25}$ und theatral bzw. audio-visuell in den Formaten von Kabarett, Comedy ${ }^{26}$, theatralen Inszenierungen ${ }^{27}$ und populären Fernsehshows ${ }^{28}$ entfalten kann. Von daher ist in jüngeren Untersuchungen davon ausgegangen worden, dass das Satirische ein Verfahren ist, welches sich im Register des Komischen entfaltet und durch Übertreibung soziale Zustände, Gruppen oder Personen als kritikwürdig, lächerlich, inakzeptabel, unerträglich oder empörend ausweist. Zu den Gattungsbezeichnungen, die eher als Verfahren zu kennzeichnen sind, gehören auch die hypertextuellen, hyperikonischen und hypermedialen Verfahren von Parodie ${ }^{29}$, Persiflage und Travestie, die ebenfalls im Register des Komischen angesiedelt sind.

Im Register des Ernsten bewegen sich dagegen die Polemik und die Streitschrift, was Ver-

21 Siehe den Beitrag von Burkhard Meyer-Sickendiek in diesem Band. Vgl. daneben Meyer-Sickendiek (2010) Theorien der Satire.

22 Zur Polemik im Rahmen der Streitkultur des 16. Jahrhunderts vgl. den Beitrag von Kai Bremer in diesem Band. Vgl. außerdem Stauffer (2003) Polemik; zur Relation Polemik und Invektive vgl. Koster (2010) Invektive und Polemik. 23 Zum Pamphlet vgl. van den Berg (2003) Pamphlet.

24 Zum Pasquill siehe den Beitrag von Gerd Schwerhoff in diesem Band.

25 Zur Karikatur vgl. den Beitrag von Lea Hagedorn in diesem Band.

26 Vgl. Koch (2015) Ethno-Comedy.

$27 \mathrm{Zu}$ invektiven theatralen Inszenierungen in der Form artivistischer Interventionen siehe den Beitrag von Lars Koch in diesem Band.

28 Vgl. Kanzler (2019) (Meta-)Disparagement Humour sowie ihren Beitrag in diesem Band.

29 Zur Bildparodie vgl. den Beitrag von Jürgen Müller in diesem Band. 
fahren der Ridikülisierung mit Anleihen im Register des Komischen aber nicht ausschließt. Beide geben sich, wie Kai Bremer in seinem Beitrag zur Streitschriften-Literatur des 16. Jahrhunderts zeigt, als reaktive Gattungen, die sich auf vorausgehendes und als empörend markiertes Handeln und Verhalten beziehen, weniger auf soziale oder politische Zustände, die als Zielscheibe satirischer Verfahren dienen. ${ }^{30}$ Schon solche mit der Behauptung einer ,Reaktion' verbundenen Bezugnahmen verweisen auf die erhebliche kommunikative Dynamik invektiver Sprech- oder Bildakte, denn sie werden nicht nur in Gattungen wie der Polemik oder der Streitschrift eingesetzt, sondern fungieren breitgefächert als Legitimation invektiver Akte. Nicht selten erfassen invektive Modalitäten auch die Formebene; sie motivieren beispielsweise ästhetische Innovationen, die sich aus einer invektiven Konstellation gegenüber den hergebrachten Ausdrucksformen ableiten lassen, wie Jürgen Müller in seinem Beitrag zur deutschen Bildparodie im 16. Jahrhundert zeigt. Die invektiven Formen korrelieren ferner mit unterschiedlichen medialen Bedingungen und Konstellationen, die eigene Darstellungsmöglichkeiten eröffnen und ausprägen, wie beispielsweise den Shitstorm. ${ }^{31}$ Insofern Invektivität in Bezug auf bestimmte kommunikative Konstellationen und Dynamiken zu analysieren ist, sind Aspekte der kommunikativen Praxis deshalb auch für die Beschreibung der Formebene zu berücksichtigen.

$\mathrm{Zu}$ Aspekten der kommunikativen Praxis gehören auch Praktiken, die der sprachlichen Aggression $^{32}$ zugeordnet, jedoch auf unterschiedlichen Ebenen rubriziert werden. Der sprachlichen Aggression zurechenbar wären auch alle Formen von hate speech, dem populärsten Konzept zur Bezeichnung invektiver Sprechakte. ${ }^{33}$ Allerdings ist - trotz der onomasiologisch-semantischen Nähe von Aggression und Hass - in der Forschung umstritten, ob hate speech affektgesteuert ist und, wenn ja, ob sie zuallererst hassindiziert oder vielmehr hassproduzierend ist. ${ }^{34}$ Wie Unter-

$30 \mathrm{Vgl}$. den Beitrag von Kai Bremer in diesem Band. $31 \mathrm{Vgl}$. Marx (2019) Von Schafen im Wolfspelz; Marx (2021) Das Dialogpotenzial von Shitstorms.

32 Vgl. die Einleitung von Silvia Bonachhi sowie die Beiträge in: Bonacchi (2017) Sprachliche Aggression.

33 Vgl. Butler (2006) Hass spricht.

34 Zur Diskussion um das Konzept hate speech vgl. die Beiträge in: Wachs / Koch-Priewe / Zick (2021) Hate Speech. suchungen zu den affektsteuernden Effekten herabsetzender populistischer Kommunikationsakte, wie etwa denen Donalds Trumps, zeigen, scheint zumindest für sie eher Letzteres zu gelten. ${ }^{35}$

Von Interesse für die Untersuchung herabsetzender Sprechakte ist nicht zuletzt die lexikalische Ebene. Neben Beleidigungen, die jenseits des Aspekts der Kränkung durch ihre rechtliche Rahmung charakterisiert werden können, ${ }^{36}$ sind dies in erster Linie Schimpfwörter, die mit bestimmten Frames verknüpft sind, wie Jan Martin Lies am Beispiel des Schimpfwortgebrauchs in den innerevangelischen Kontroversen der zweiten Hälfte des 16. Jahrhunderts zeigt. Schwerer identifizierbar sind dagegen die in verbreiteten Sprachgebrauchsmustern vorgenommenen herabsetzenden Prädikationen und Präsuppositionen, die - anders als Schimpfwörter oder Ethnophaulismen ${ }^{37}$ - an ihrer Oberfläche nicht unmittelbar als schmähend erkennbar bzw. pseudo-wissenschaftlich verbrämt sein können, wie Anja Lobenstein-Reichmann in ihrem Beitrag zur sprachlichen Konstruktion von ,Rasse' zeigt. ${ }^{38}$

$\mathrm{Zu}$ bedenken ist des Weiteren das Verhältnis der unterschiedlichen invektiven Formen und Muster zueinander: Wie etwa ist die Beziehung von etablierten Gattungen, die Modalitäten der Herabsetzung bereits inkludieren, zu neuen invektiven Gattungen zu denken? Was unterscheidet etwa die Schmährede und die Wutrede?

Siehe daneben auch die Beiträge in: Meibauer (2013) Hassrede sowie aus linguistischer Sicht Marx (2018) Hate Speech. Einen aufschlussreichen Beitrag liefert daneben Scharloth (2017) Hassrede und Invektivität, der die Bezichtigung einer Äußerung als Hassrede aus invektivitätstheoretischer Perspektive als metainvektive Diskursintervention deutet, die selbst Teil invektiver Kommunikation ist.

35 Vgl. Koch / Nanz / Rogers (2020) The Great Disruptor, bes. S. 6-8.

$36 \mathrm{Zu}$ den juristischen Aspekten vgl. den Kommentar von Hilgendorf (2009) Beleidigung; zu den gesprächsrhetorischen Aspekten siehe Meier (2021) Beleidigungen.

$37 \mathrm{Vgl}$. Tenchini (2017) Multi-Akt-Semantik. Den Begriff des Ethnophaulismus als abwertende Bezeichnung für eine ethnisch oder rassistisch definierte Gruppe hat der amerikanische Psychologe Abraham Aron Roback geprägt; vgl. Roback (1944) A Dictionary.

$38 \mathrm{Vgl}$. dazu die Beiträge von Jan-Martin Lies und Anja Lobenstein-Reichmann in diesem Band. Siehe daneben die Beiträge in: Hornscheidt (2011) Schimpfwörter. Grundlegend auch: Lobenstein-Reichmann (2013) Sprachliche Ausgrenzung; von unschätzbarer Genauigkeit: Klemperer (1947) LTI. 
Handelt es sich hier lediglich um einen Begriffstransfer, geht damit auch ein konzeptioneller Transfer einher oder handelt es sich um Gattungsbezeichnungen, die nichts miteinander zu tun haben? Welche Gattungen erweisen sich als besonders anschlussfähig für invektive Akte (als , invektivierbar')? Welche Rolle spielen invektive Verfahren (also etwa das Sarkastische, das Ironische) als Modalitäten im poetologischen Sinne? ${ }^{39}$ Inwiefern lassen sich invektive Gattungen über Mündlichkeit, Schriftlichkeit und Bildlichkeit differenzieren? Welche Affekt- und Emotionsregime etablieren sie dabei jeweils? Wie ist der Formwandel des Invektiven, etwa das Verhältnis zwischen vormodernen und modernen beziehungsweise zeitgenössischen invektiven Gattungen zu denken und zu beschreiben? Wie der Beitrag von Lea Hagedorn zeigt, lässt er sich nicht zuletzt an der Transformation von Gattungsbegriffen wie dem der Karikatur erkennen. In welcher Weise invektive Modalitäten ästhetische Formen zunehmend prägen, entwickelt der Beitrag von Lars Koch, der sich den artivistischen Interventionen widmet, mit denen Christoph Schlingensief und das Zentrum für politische Schönheit gesellschaftliche Diskurse und Dispositive und ihre invektiven Effekte reflektieren und dabei zugleich invektieren.

Wie die nachfolgenden Untersuchungen insgesamt verdeutlichen, lassen sich vielfältige Austauschprozesse beobachten, in denen einerseits rhetorisch-literarische Gattungen alltagssprachliche Elemente integrieren und bearbeiten, andererseits in der Alltagskommunikation ästhetische Schemata angeeignet und transformiert werden. Etablierte literarische und rhetorische Gattungen fungieren damit ebenso als Formarchive, die Muster der Herabsetzung in der Alltagskommunikation verfügbar halten, wie umgekehrt Muster der Alltagskommunikation in solchen Gattungen aufgenommen, iteriert und transformiert werden.

Deshalb fokussiert der Band mit den invektiven Gattungen als Untersuchungsbereich ein Schnittfeld, das rhetorische, literarische und bildkünstlerische Gattungen sowie kommunikative Gattungen im Sinne mehr oder minder fest etablierter Sprachgebrauchsmuster und auch die

39 Derartige Fragen erörtert der Beitrag von Burkhard Meyer-Sickendiek, der die spezifischen Ausprägungen des Satirischen in der Moderne unter poetologischen Gesichtspunkten untersucht. kommunikativen Affordanzen medialer Formate erfasst. Die Fragestellung richtet sich zum einen auf den Formaspekt des Invektiven, zum anderen auf die Effekte von Invektivität für die Konstitution und Transformation unterschiedlicher Gattungen. Übergreifend thematisieren die Beiträge die Frage, welche Bedeutung einerseits den Gattungen für die Performanz, Redundanz und Varianz invektiver Rede zukommt, welche Rolle invektive Kommunikation andererseits für die Transformation und Genese von Gattungen beziehungsweise von Sprachgebrauchsmustern spielt und wie Gattungen und Muster wiederum auf die invektiven Dynamiken zurückwirken. Dass die hier behandelten Gattungen und Modalitäten des Invektiven nicht erschöpfend sein können, versteht sich angesichts der Dynamik invektiver Kommunikation nahezu von selbst. Kennzeichnend für invektive wie metainvektive Kommunikation ist überdies, dass sie sämtliche literarischen, rhetorischen und kommunikativen Gattungen integrieren und transformieren kann und nicht an eine Liste invektiver Gattungen gebunden ist, auch wenn sich diese für herabsetzende Kommunikationsakte anbieten und dementsprechend häufig genutzt werden.

\section{Literaturverzeichnis}

Ayaß, Ruth (2011): Kommunikative Gattungen, mediale Gattungen. In: Habscheid, Stephan (Hg.): Textsorten, Handlungsmuster, Oberflächen. Berlin/ Boston: De Gruyter, S. 275-295.

Berg, Hubert van den (2003): Pamphlet. In: Historisches Wörterbuch der Rhetorik 6: Must - Pop. Tübingen: Niemeyer, Sp. 488-495.

Bonacchi, Silvia (Hg.) (2017): Verbale Aggression. Multidisziplinäre Zugänge zur verletzenden Macht der Sprache. Berlin/Boston: De Gruyter.

Bubenhofer, Noah (2009): Sprachgebrauchsmuster. Korpuslinguistik als Methode der Diskurs- und Kulturanalyse. Berlin/New York: De Gruyter.

Butler, Judith (2006): Hass spricht. Zur Politik des Performativen, Frankfurt a. M.: Suhrkamp.

Dürscheid, Christa (2013): Medien, Kommunikationsformen, kommunikative Gattungen. Linguistik Online, 22/1, S. 3-16. URL: https://doi.org/10.13092/ lo.22.752 (letzter Zugriff: 30.08.2021).

Dürscheid, Christa/Frick, Karina (2016): Schreiben digital: Wie das Internet unsere Alltagskommunikation verändert. Stuttgart: Kröner.

Erler, Michael/Tornau, Christian (2019): Einleitung. Was ist antike Rhetorik. In: Erler, Michael/Tornau, 
Christian (Hg.): Handbuch antike Rhetorik, Berlin/ Boston: De Gruyter, S. 1-16.

Fox, Richard/Panagiotopoulos, Diamantis/Tsouparopoulou, Christina (2015): Affordanz. In: Ott, Michael R./Sauer, Rebecca/Meier, Thomas (Hg.): Materiale Textkulturen. Konzepte - Materialien - Praktiken. Berlin/Boston/ München: De Gruyter, S. 63-70.

Genette, Gérard (1993): Palimpseste. Die Literatur auf zweiter Stufe. Aus dem Französischen von Wolfram Bayer und Dieter Hornig. Frankfurt a. M.: Suhrkamp [Paris 1982].

Genette, Gérard (2001): Paratexte. Das Buch vom Beiwerk des Buches. Mit einem Vorwort von Harald Weinrich. Aus dem Französischen von Dieter Hornig. Frankfurt a. M.: Suhrkamp [Paris 1987].

Günthner, Susanne/Knoblauch, Hubert (1996): Die Analyse kommunikativer Gattungen in Alltagsinteraktionen. In: Michaelis, Susanne/Tophinke, Doris (Hg.): Texte - Konstitution, Verarbeitung, Typik. München: LINCOM Europa, S. 35-57.

Günthner, Susanne (1995): Gattungen in der sozialen Praxis. In: Deutsche Sprache 3, S. 193-218.

Günthner, Susanne/Knoblauch, Hubert (1994): „Forms are the Food of Faith". Gattungen als Muster kommunikativen Handelns. In: Kölner Zeitschrift für Soziologie und Sozialpsychologie 46, S. 693-723.

Gymnich, Marion (2010): Gattung und Gattungshistoriographie. In: Zymner, Rüdiger ( $\mathrm{Hg}$.$) : Handbuch$ Gattungstheorie. Stuttgart/Weimar: Metzler, S. $131-158$.

Helmrath, Johannes (2010): Streitkultur. Die "Invektive" bei den italienischen Humanisten. In: Laureys, Marc/ Simon, Roswitha (Hg.): Die Kunst des Streitens. Inszenierung, Formen und Funktionen öffentlichen Streits in historischer Perspektive. Göttingen: V\&R unipress, S. 259-294.

Hess-Lüttich, Ernest W. B. (Hg.) (2021): Handbuch Gesprächsrhetorik. Berlin/Boston: De Gruyter.

Hilgendorf, Eric (2009): § 185. In: Laufhütte, Heinrich (Hg.): Leipziger Kommentar zum Strafgesetzbuch. 12. Aufl. Bd. 6: §§ 146-210. Berlin: De Gruyter, S. $1229-1348$.

Jauß, Hans Robert (1977): Theorie der Gattungen und Literatur des Mittelalters. In: Alterität und Modernität der mittelalterlichen Literatur. Gesammelte Aufsätze 1956-1976. München: Fink, S. 327-358.

Kanzler, Katja (2019): (Meta-)Disparagement Humour: The Poetics and Politics of Mockery in the Sitcom Two Broke Girls. In: Hägi-Mead, Sara/Flubacher, Mi-Cha (Hg.): Taboo and Transgression. Dresden: Theoretische Beiträge des Zentrums für Integrationsstudien, S. 15-24.

Klemperer, Victor (1947): LTI. Notizbuch eines Philologen. Leipzig: Aufbau.

Koch, Lars (2015): Die Ethno-Comedy - Lachen an den Rändern der ,Leitkultur'. In: Neuhaus, Stefan (Hg.): Das Komische in der Kultur. Frankfurt a. M./New York: Campus, S. 465-481.

Koch, Lars/Nanz, Tobias/Rogers, Christina (2020) (Hg.): The Great Disruptor. Eine Annäherung. In: Dies.
(Hg.): The Great Disruptor. Über Trump, die Medien und die Politik der Herabsetzung. Stuttgart: J. B. Metzler, S. 1-19.

Koster, Severin (2010): Invektive und Polemik in der Antike. Versuch einer Verhältnisbestimmung. In: Wischmeyer, Oda/Scornaienchi, Lorenzo (Hg.): Polemik in der frühchristlichen Literatur. Texte und Kontexte. Beihefte zur Zeitschrift für die neutestamentliche Wissenschaft und die Kunde der älteren Kirche 170. Berlin/Boston: De Gruyter, S. 39-53.

Koster, Severin (1980): Die Invektive in der griechischen und römischen Literatur. Meisenheim am Glan: Anton Hain.

Hornscheidt, Antje Lann (Hg.) (2011): Schimpfwörter Beschimpfungen - Pejorisierungen: Wie in Sprache Macht und Identitäten verhandelt werden. Frankfurt a. M.: Brandes \& Apsel.

Lobenstein-Reichmann, Anja (2013): Sprachliche Ausgrenzung im späten Mittelalter und in der frühen Neuzeit. Berlin/Boston: De Gruyter.

Lomborg, Stine (2011): Social media as communicative genres. In: MedieKultur. Journal of media and communication research 27/51, S. 55-71.

Lomborg, Stine (2014): Social Media, Social Genres. Making Sense of the Ordinary. New York/Oxon: Routledge.

Marx, Konstanze (2017): Rekontextualisierung von Hate Speech als Aneignungs- und Positionierungsverfahren in Sozialen Medien. In: Aptum 13/2, S. 132-146.

Marx, Konstanze (2018): Hate Speech - ein Thema für die Linguistik. In: Albers, Marion/Katsivelas, Ioannis ( $\mathrm{Hg}$. ): Recht \& Netz. - Baden-Baden: Nomos, S. 37-57.

Marx, Konstanze (2019): Von Schafen im Wolfspelz - Shitstorms als Symptome einer medialen Emotionskultur. In: Hauser, Stefan/Luginbühl, Martin/ Tienken, Susanne (Hg.): Mediale Emotionskulturen. Bern: Lang, S. 135-153.

Marx, Konstanze (2021): Das Dialogpotenzial von Shitstorms. In: Hess-Lüttich, Ernest W. B. (Hg.): Handbuch Gesprächsrhetorik. Berlin/Boston: De Gruyter, S. 409-427.

Meier, Simon/Marx, Konstanze (2019): Doing Genre in the Digital Media. In: Brock, Alexander/Pflaeging, Jana/Schildhauer, Peter (Hg.): Genre Emergence. Developments in Print, TV and Digital Media. Frankfurt a. M./New York: Lang, S. 191-212.

Meier, Simon (2021): Beleidigungen als Gegenstand der Gesprächsrhetorik. In: Hess-Lüttich (Hg.): Handbuch Gesprächsrhetorik. Berlin/Boston: De Gruyter, S. 389-408.

Meyer-Sickendiek, Burkhard (2010): Theorien der Satire. In: Zymner, Rüdiger (Hg.): Handbuch Gattungstheorie. Stuttgart/Weimar: Metzler, S. 331-334.

Münkler, Marina (2015): Legende/Lügende. Die protestantische Polemik gegen die katholische Legende und Luthers Lügend von St. Johanne Chrysostomo. In: Schwerhoff, Gerd/Piltz, Eric (Hg.): Gottlosigkeit und Eigensinn. Religiöse Devianz im konfessionellen Zeitalter. Berlin (Beiheft zur Zeitschrift für Historische Forschung, 51), S. 121-147. 
Roback, Abraham Aron (1944): A Dictionary of International Slurs (Ethnophaulisms). With a Supplementary Essay on Aspects of Ethnic Prejudice. Cambridge MA: Sci-Art Publishers.

Sablotny, Antje (2019): Metalegende. Die protestantische Lügende als invektive Metagattung. In: Beiträge zur mediävistischen Erzählforschung 2, S. 148-200. DOI: https://doi.org/10.25619/BmE2019228 (letzter Zugriff: 01.11.2021).

Scharloth, Joachim (2017): Hassrede und Invektivität als Gegenstand der Sprachwissenschaft und Sprachphilosophie: Bausteine zu einer Theorie des Metainvektiven. In: Aptum 2, S. 116-132.

Schulz, Verena (2019): Was ist rhetorische Wirkung? Zum Verhältnis von ,Logos', ,Pathos' und ,Ethos'. In: Erler, Michael/Tornau, Christian (Hg.): Handbuch antike Rhetorik. Berlin/Boston: De Gruyter, S. 557-580.

Stauffer, Hermann (2003): Polemik. In: Historisches Wörterbuch der Rhetorik 6: Must - Pop. Tübingen: Niemeyer, Sp. 1403-1415.

Tenchini, Maria Paola (2017): Zur Multi-Akt-Semantik der Ethnophaulismen. In: Bonacchi, Silvia (Hg.): Verbale Aggression. Multidisziplinäre Zugänge zur verletzenden Macht der Sprache. Berlin/Boston: De Gruyter, S. 245-268.
Voßkamp, Wilhelm (1977): Gattungen als literarischsoziale Institutionen. In: Hinck, Walter ( $\mathrm{Hg}$.$) :$ Textsortenlehre - Gattungsgeschichte. Heidelberg: Quelle \& Meyer, S. 27-42.

Voßkamp, Wilhelm ( $\left.{ }^{9} 2004\right)$ : Gattungen. In: Brackert, Helmut/Stückrath, Jörn ( $\mathrm{Hg}$.): Literaturwissenschaft. Ein Grundkurs. Reinbek bei Hamburg: Rowohlt, S. $253-269$.

Wachs, Sebastian/Koch-Priewe, Barbara/Zick, Andreas (Hg.) (2021): Hate Speech - Multidisziplinäre Analysen und Handlungsoptionen. Theoretische und empirische Annäherungen an ein interdisziplinäres Phänomen. Wiesbaden: Springer 2021.

Zillien, Nicole (2008): Die (Wieder-)Entdeckung der Medien. Das Affordanzkonzept in der Mediensoziologie. In: Sociologia Internationalis 46/2, S. $161-181$.

Zymner, Rüdiger (2007): Gattungsvervielfältigung: Zu einem Aspekt der Gattungsdynamik. In: Gymnich, Marion/Neumann, Birgit/Nünning, Ansgar (Hg.): Gattungstheorie und Gattungsgeschichte. Trier: WVT, S. $101-116$.

Zymner, Rüdiger (2003): Gattungstheorie. Probleme und Positionen der Literaturwissenschaft. Paderborn: mentis. 